\title{
Development of a Friction Welded Joint for Future Industrial Application
}

\author{
Nadine Schubert ${ }^{1, a^{*}}$, Andreas Sterzig ${ }^{1, b}$, Reinhard Mauermann ${ }^{1, c}$, \\ Sören Hilbers ${ }^{2, \mathrm{~d}}$, Peter Kolbe ${ }^{3, \mathrm{e}}$ and Christian Kuhn ${ }^{3, \mathrm{f}}$ \\ ${ }^{1}$ Fraunhofer-Institut für Werkzeugmaschinen und Umformtechnik IWU, Reichenhainer Straße 88, \\ 09126 Chemnitz, Germany \\ ${ }^{2}$ Simufact Engineering GmbH, Tempowerkring 19, 21079 Hamburg, Germany \\ ${ }^{3}$ Gesenkschmiede Schneider GMBH, Ulmer Str. 112, 73431 Aalen, Germany \\ aNadine.Schubert@iwu.fraunhofer.de, bAndreas.Sterzig@iwu.fraunhofer.de \\ 'Reinhard.Mauermann@iwu.fraunhofer.de, dsoeren.hilbers@simufact.de, \\ ePeter.Kolbe@gsa-aalen.de, fChristian.Kuhn@gsa-aalen.de \\ ${ }^{*}$ corresponding author
}

Keywords: Friction welding, simulation, finite element method

\begin{abstract}
Ship transport continues to be the most economical intercontinental means of transportation, which is becoming more and more under observation due to increasing international environmental protection regulations. In order to protect the marine biotope, the government is introducing stricter exhaust emission regulations, which mean the further development of lowemission large engines and thus make the development of new combustion chamber components indispensable. This paper presents FE simulations of a friction welding process for two metallic materials, which are being developed as part of a BMWi joint project for the production of innovative combustion chamber components. In addition, the first results of the simulations and their comparison with real tests are presented.
\end{abstract}

\section{Introduction}

Welding is still one of the most important processes within the entire joining technology. In addition to unsurpassed cost-effectiveness, it allows constructive designs that to a large extent take into account the requirements for flexibility and weight optimization. Welding is the joining or coating of workpieces in liquid or plastic state using energy in the form of heat and/or force and movement, without or with filler materials. In principle, three classification criteria apply to welding processes:

- purpose (connecting or applying)

- connection mechanism (melting, pressing, reaming)

- execution type (manual, mechanized, automated)

Friction welding is at the forefront of current investigations for the production of various combustion chamber components in the design of a composite material. Due to the increased material price of the nickel-base alloys, which will be analyzed later in the course of the investigations, the investigations of the friction welding process with conventional piston materials are presented in this paper $(42 \mathrm{CrMo} 4,38 \mathrm{MnVs} 6)$. In contrast to the previously known friction welding process, in which heat is generated during the actual joining process due to friction of the components to be joined, the aim of the investigations presented here is to reduce the process time by means of an upstream partial heating. The connection itself is created by heating up both components due to friction until they have a dough-like consistency. The simultaneously acting pressure creates a material-locking connection between the two components. The special feature of friction welding processes is that the joining process itself takes place below the melting temperature of the components. This, in turn, is one of the greatest challenges, which means that the optimal process window must be identified using FE simulations [1, 5]. 
The present comparison of the two friction welding variations was made with the objective of highlighting the advantages of preheated components for friction welding compared to the conventional procedure. Since friction welding without preheating represents the state of the art, it is important to consider not only the process differences, but also the influence of the changed temperature on the welding result. In this way, the usability of preheated blanks for the production of friction welding components is to be demonstrated and the process is to be presented as an alternative manufacturing process for future friction welding joints and at best expanded as a result of further modifications.

The publication is structured as follows: at the beginning a short insight into the friction welding process is given. Following this, the potential of the FE simulation software used is presented on the basis of a concrete reference geometry (Fig. 1). A comparison between the FE simulations carried out and the real tests carried out will show how simulations can serve as a tool for process and geometry optimization. Finally, an outlook on future applications of the FE module in connection with further material combinations is given.

\section{Friction Welding}

According to DIN 8580, joining is defined as "the permanent joining or other joining of two or more workpieces of geometrically determined solid form or of such workpieces with formless material; the cohesion is created locally and increased as a whole" [2].

One such joining process is friction welding, in which two or more parts are joined together to form a continuum. To ensure this, the workpieces to be joined are manufactured using heat and/or force with or without filler material. After a material- and component-specific speed and friction time, the rotation is stopped and the two parts are brought together linearly using a surface-related friction force. A welding bead is formed, which is often turned off immediately after the end of the welding process, but can also be left as long as the installation dimensions permit. Many material pairs that are not suitable for fusion welding can be joined by friction welding as a joining process in solid phase without melt, since the joining temperature is below the melting temperature of the materials and thus the deformation in conjunction with the heat introduced leads to a fine-grained structure in the area of the recrystallization temperature. There are a number of different advantages that argue in favour of using the friction welding process. For example, many material combinations can be welded which are not suitable for fusion welding, it can be fully mechanised with a high degree of repeatability, both solid and hollow cross-sections can be used, no welding filler is required, and a fine-grained, recrystallized structure is formed. On the disadvantage side, there are only a few points to mention that are usually component or machine-related. Both the diameters and the lengths of the components are limited by the machine size, whereby the size of the machine is usually limited by the high purchase costs of these. During the welding process bead formation occurs, which in most cases makes reworking indispensable [1, 2, 3].

With regard to the advantages and disadvantages of this method, the benefit of partial preheating of the bodies to be joined is to be investigated. For example, partial heating of the joining partners should result in a reduction of the process time.

\section{Experimental and Numerical Investigations}

With regard to the planned future design of friction welding, experimental investigations and FE simulations were carried out for two variants of friction welding. A conventional welding process, hereinafter referred to as "Variant 1", should be compared with a preheated reference geometry ("Variant 2"). Both variants were tested with the quenched and tempered steel 42CrMo4 (Chemical composition of $42 \mathrm{CrMo} 4$ - as seen in table 1). For verification of the results, both the conventional process and the modified process were performed on an experimental and numerical basis using commercial software. 
Table 1: Chemical composition of 42CrMo4

\begin{tabular}{lllllllll}
\hline Composition in wt.-\% & $\mathrm{C}$ & $\mathrm{Cr}$ & $\mathrm{Si}$ & $\mathrm{Mo}$ & $\mathrm{Ni}$ & $\mathrm{Cu}$ & $\mathrm{Mn}$ & $\mathrm{Fe}$ \\
\hline 42CrMo4 & 0.41 & 1.03 & 0.20 & 0.21 & 0.18 & 0.31 & 0.62 & balance \\
\hline
\end{tabular}

\section{Experiments}

For the comparability of the tests, the following reference geometry was selected for both components to be connected (Fig. 1). The geometry was used to make initial statements regarding the process parameters. As a result of heating, this was essential as the modified technology differs greatly from the conventional process.
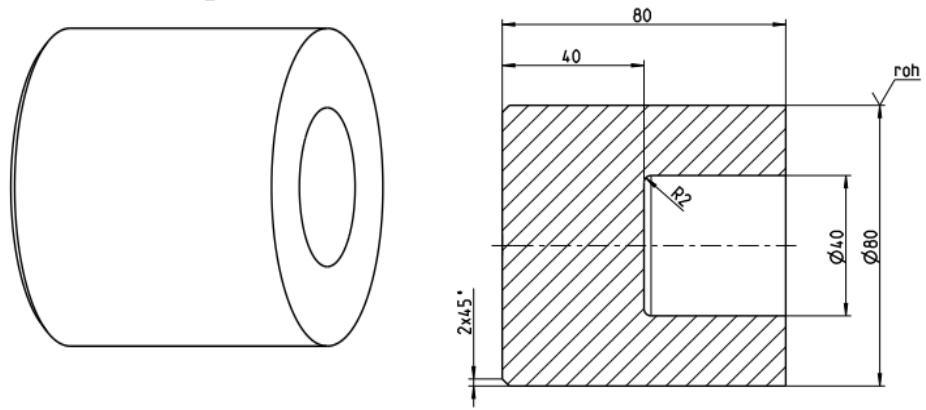

Fig. 1: Reference geometry used for the welding tests (dimensions in $\mathrm{mm}$ )

The conventional friction welding process of variant 1 was carried out by positioning the two parts to be joined in the friction welding machine. As a result of the high speed and the compression pressure, the two bodies were finally joined together. Variant 2 was implemented by heating the two references by means of a gas burner, so that the front surfaces were heated to approx. $550-600{ }^{\circ} \mathrm{C}$. After heating, the two bodies were positioned in the friction welding machine and welded together. The machine used was the KUKA RS 80, which is located at the die forging company Schneider $\mathrm{GmbH}$ in Aalen. The following Fig. 2 shows an example of the friction welding process. The blue line indicates the spindle speed $(1200 \mathrm{rpm})$, the black line indicates the pressure across the path (red line). After a process time of $26 \mathrm{~s}$ the complete welding is finished.

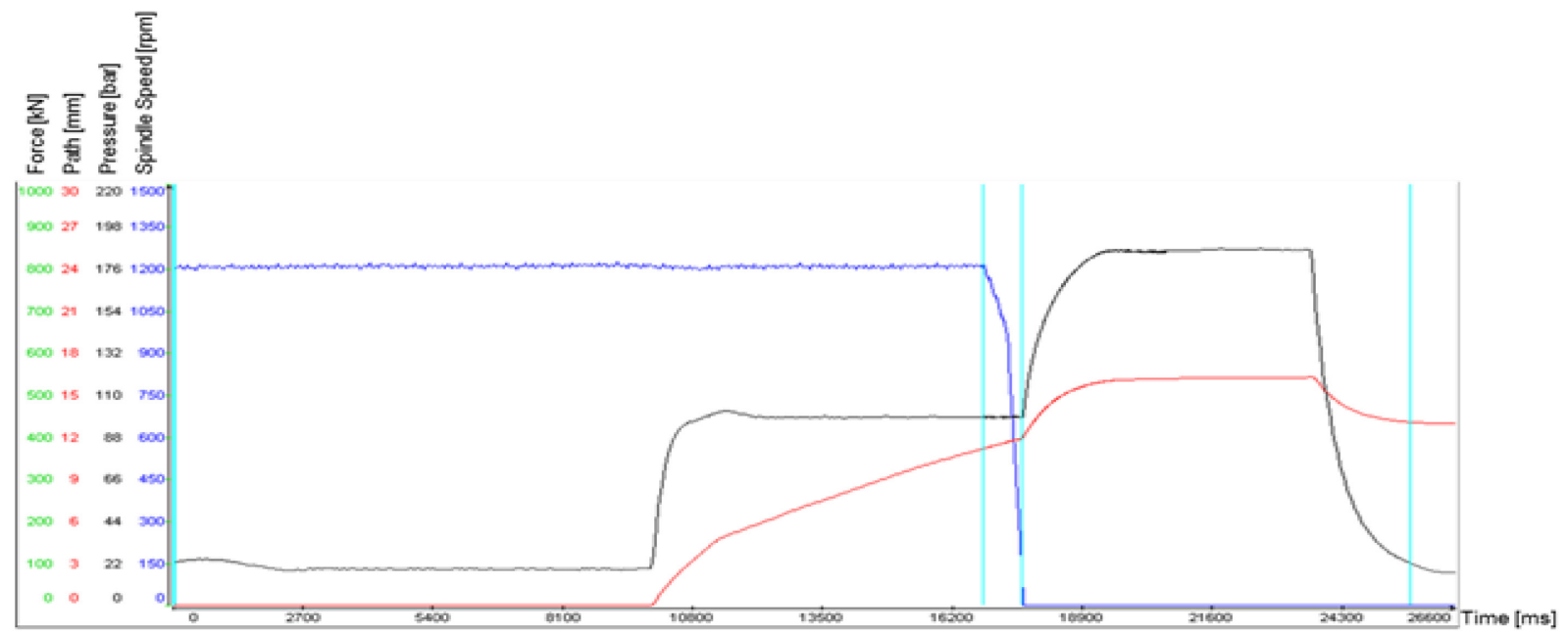

Fig. 2: Exemplary sequence of a friction welding process (42CrMo4)

\section{Numerical Representation of Friction Welding Processes}

Simufact Forming 15.0 is used for the simulation of the friction welding processes. According to the comparability the described reference geometry was also used in the FE investigations. The processes are modeled as 2D axial symmetrical simulations with isotropic elastic-plastic material behavior. The temperature distribution is described using finite elements and calculated using 
transient heat conduction and the finite difference method. The mechanical behavior is calculated using the incremental finite element method. Both, thermal and mechanical simulations are coupled for every time step.

Friction welding processes can be simulated with two different approaches. The first approach uses directly the heat generated by the applied friction law and its friction coefficients. This method underestimates the generated heat. In order to get a reasonable temperature distribution unphysically high friction coefficients need to be defined. One reason for this behavior could be that the conventional friction laws like Coulomb or shear friction law are not valid for the material state near melting temperature. Coulomb and shear friction law are developed for dry friction as well as mixed friction of solid bodies in a certain temperature range. During a friction welding process the material becomes dough like and does not represent a solid body anymore. Furthermore the time step size needs to be very small in order to capture the movement of the joint partners and the generated heat. This results in a time expensive simulation. Thus the first approach is not applicable and recommended. The second approach uses an analytical model to introduce a heat flux on the contact nodes. The model is based on a user-defined rotational velocity, a user-defined friction coefficient, the contact normal stress and the distance of the contact nodes from the rotation axis. The friction coefficient also contains the efficiency of the energy conversion from mechanical into heat energy. Using this information a heat flux is calculated and equally applied on the corresponding contact node. This model allows an easy calibration of the friction coefficient and also a tabular definition of the friction coefficient with respect to the temperature. The tabular definition can be used to limit the heat generation to a certain temperature. For variant 2, the local heating of the reference bodies had to be taken into account. To make this relatively trivial, a simple local heating calculation was prefaced in Simufact. The partial pre-heating of the geometries is simulated using heat conduction in combination with a near contact tolerance. This approach allows a free expansion of the components without introducing stresses. When the near contact tolerance is used, the bodies do not need to be in real mechanical contact in order to conduct the heat across the contact area. Contact is only assumed for the thermal calculation with a defined heat transfer coefficient. The near contact tolerance defines the maximum distance two bodies can have to each other in which the heat transfer acts. Bodies outside the near contact tolerance will be neglected for the thermal heat transfer. Using this approach a defined temperature profile can be created inside the geometries with a minimum effort in modeling and a short simulation time $[4,6]$.

\section{Results and Discussion}

As already explained, a conventional friction welding process was compared with a preheated component for the investigations. First, the results of the conventional process are analysed in more detail. The FE-model used for this is shown in Fig. 3 below. For the friction welding process itself, the two bodies to be joined are required and a support plate for each. This plate is used to transfer the kinematics, as the upper body simultaneously travels a defined compression path (Fig. 2) at a corresponding speed.
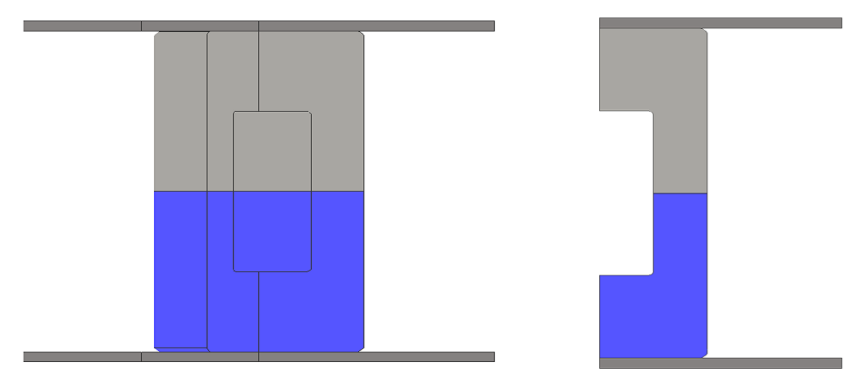

Fig. 3: FE-model of the friction welding process for both variants

With regard to the calculation times, the simulations were calculated as 2-dimensional models. The following Fig. 4 shows the comparison between the real test and the FE simulation. 


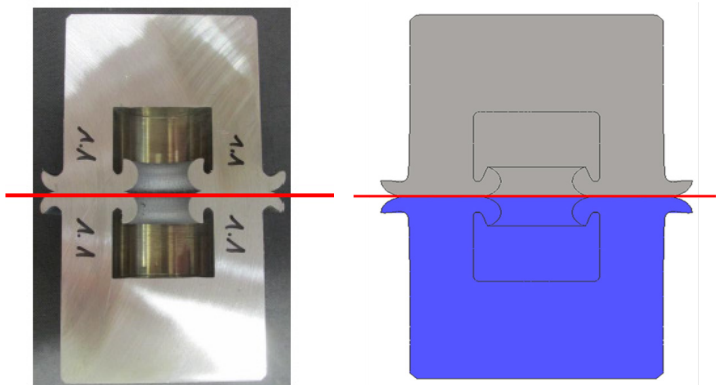

Fig. 4: Standard friction welding result - left: real test, right: FE-simulation

It can clearly be seen that the formation is similar to the bead. In both tests, the bead inside the component forms much more strongly than on the surface of the shell. The dimensions of the joined bodies are also relatively similar. The total height of the real sample after the joining process was $149.21 \mathrm{~mm}$ and that of the simulation was $148.5 \mathrm{~mm}$. For the experiments of variant 2 , the end faces to be joined were partially heated until they had a temperature of approx. $500-600{ }^{\circ} \mathrm{C}$. Subsequently, the same program sequence of the welding process as for variant 1 took place. The partial heating in Simufact was modelled with a replacement body for the coil. In the following Fig. 5 the coil can be seen in the middle between the two joining partners. With the help of the described close contact tolerance, this heated the joint before the actual joining process.
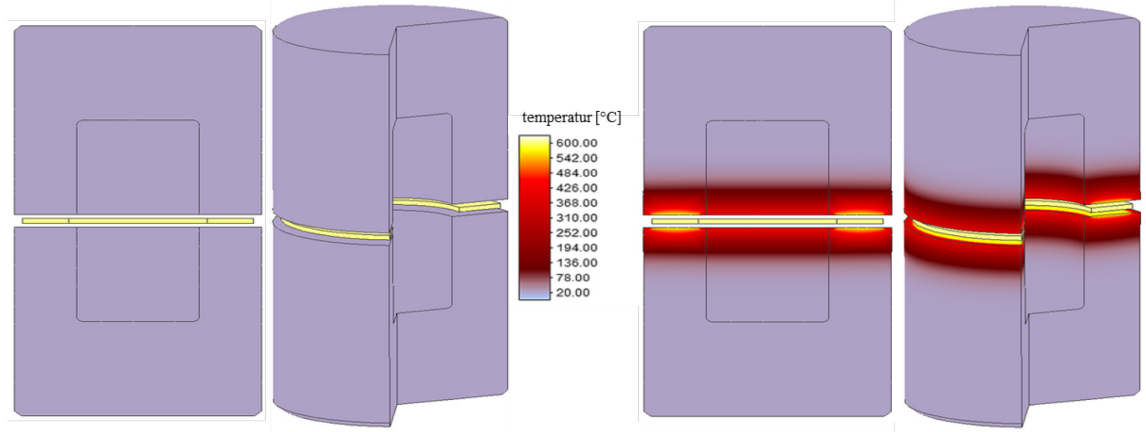

Fig. 5: Preheating left: Initial condition $\left(20^{\circ} \mathrm{C}\right)$, right: Heating result

In the following comparison of the two joined components it becomes clear, as in variant 1 , that the simulated weld bead has a similar formation as that from the real test. The final height of the component is also similar. The height of the real part is $133.37 \mathrm{~mm}$ and that of the simulated component $136.6 \mathrm{~mm}$.
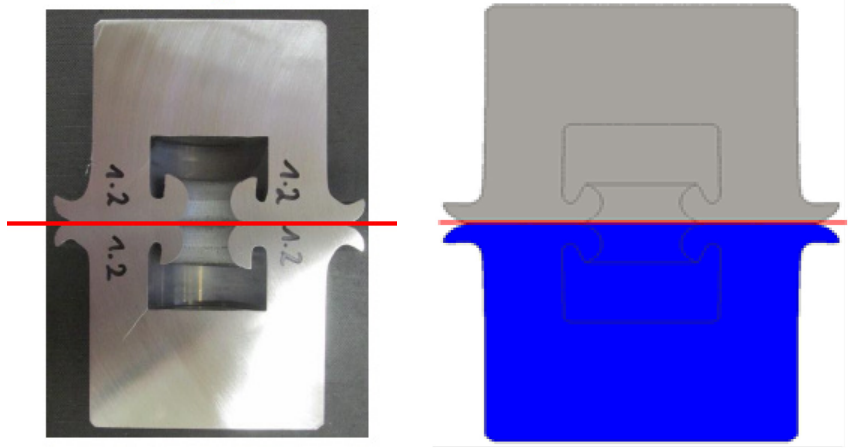

Fig. 6: Friction welding with preheating left: real test, right: FE-simulation result

With the help of preheating, the process time of variant 2 could be reduced to 12.9 seconds compared to variant 1 with 22.7 seconds. Finally, due to the real tests and the use of the finiteelement method, not only a reduction of the process time due to preheating could be confirmed, but also the use of the simulation software for the design of friction welding components could be proven. 


\section{Summary and Prospects}

Scientific and technological questions in the field of joining of cost-intensive materials were the starting point for the more precise considerations of the conventional friction welding process. As a result of local preheating of the end faces, the same joining results should be achieved with a shortened process time. The process time of variant 2 could be reduced to 13 seconds compared to variant 1 with 23 seconds. Due to the expensive materials, tests were initially carried out with conventional quenched and tempered steel (42CrMo4) as described. Based on the verification of the results of the real tests with those of the FE simulations, it became clear that numerical models (with and without preheating) are suitable for making early statements on the joint connection. In the further course of the investigations, the experiments are to be extended to other materials, especially nickel-based alloys. As soon as these are to be joined without defects, a composite of different materials is striven for. To illustrate the FE calculations, the real tests will be carried out in the further course of the project with inductive heating.

\section{Acknowledgments}

Supported by:

貌 Federal Ministry for Economic Affairs and Energy

on the basis of a decision by the German Bundestag
The Federal Ministry of Economics and Technology (BMWi) sponsor the research project with the program "Maritime technologies of the next generation". This work is the result of the collaboration with the TU Bergakademie Freiberg Institut für Werkstofftechnik and Gießerei-Institut, Fraunhofer IWU Chemnitz, Gesenkschmiede Schneider Aalen, MET Motoren- und Energietechnik Rostock and Wissenschaftlich-Technisches Zentrum Roßlau.

\section{References}

[1] N. Bay, Cold Welding, ASM Handbook, Volume 6A, Welding Fundamentals and Processes, 2011, pp 711-716.

[2] J. Ruge, Handbuch der Schweißtechnik: Band II - Verfahren und Fertigung, Springer-Verlag Berlin Heidelberg GmbH, 1993.

[3] H. Behnisch, Kompendium Schweißtechnik, Band 1: Verfahren der Schweißtechnik, Düsseldorf: DVS-Verlag, 2002.

[4] Information on https://www.konstruktionspraxis.vogel.de/simulationssoftware-simufactforming-14-deckt-jetzt-zusaetzliche-fertigungsverfahren-ab-a-582399/

[5] M. Lotz, Technische Universität München, Erhöhung der Fertigungsgenauigkeit beim Schwungrad-Reibschweißen durch modellbasierte Regelungsverfahren, Herbert UTZ Verlag Wissenschaft, 2013.

[6] Information on http://www.tl-ing.de/data/ueber_uns/veroeffentlichungen/SysweldForum09_ TROMMER_Loose_et_al.pdf(last accessed 10 December 2018) 